Die Förderung von Umweltmanagementsystemen als Baustein von Umweltallianzen

\title{
Südtirol auf Bayerns Spuren
}

\section{Für eine nachhaltige Entwicklung bedarf es neuer Instrumente im Verhältnis zwischen Staat und gesellschaftlichen Gruppen. Der Wirtschaft kommt hier eine zentrale Bedeutung zu. Ein häufiges Instrument sind so genannte Umwelt- pakte. Wie die Beispiele Bayern und Südtirol zeigen, spielt der Ausbau von Umweltmanagementsystemen dabei eine entscheidende Rolle.}

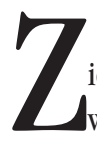
Von Guiseppe Santillo iel jedes Umweltpakts ist verstärkter Umweltschutz auf der Grundlage von Freiwilligkeit, Eigenverantwortung und Kooperation. In Deutschland den Anfang machte hier Bayern im Jahr 1995 (1). Andere Beispiele sind Umweltallianzen in Berlin (1998, Chemie- und Elektrobranche) und Sachsen (2002). In Brandenburg, Baden-Württemberg und Nordrhein-Westfalen werden ähnliche Initiativen vorbereitet.

Die meisten Umweltvereinbarungen in den deutschen Bundesländern beschränken sich im Wesentlichen auf Umweltmanagement und Verwaltungsvereinfachung. Im Unterschied dazu sind im vor eineinhalb Jahren erneuerten Umweltpakt Bayern zwischen bayerischer Wirtschaft und Staatsregierung Ziele, Gemeinschaftsprojekte und Umsetzungsmaßnahmen in insgesamt 11 Themenfeldern vereinbart worden. Hierzu stehen teilweise Förderprogramme zur Verfügung, außerdem ist ein regelmäßiges Controlling vorgesehen. Beispiele sind die Verringerung von Treibhausgasemissionen, Ressourcenschonung, integrierte Produktpolitik (2), umweltverträgliche Mobilität sowie die Förderung von Umweltmanagementsystemen (UMS).

Unternehmen, die ein Managementsystem nach EMAS einführen, erhalten Erleichterungen beim Verwaltungsvollzug durch die Staatsregierung. Klei-

ne und mittlere Unternehmen (KMU) können aus dem Umweltberatungs- und Auditprogramm gefördert werden (3). Durch die Förderung sollen KMU $\mathrm{zu}$ freiwilligen, kontinuierlichen Verbesserungen des betrieblichen Umweltschutzes ermutigt werden. Seit 1995 wurden aus diesem Programm ca. 1500 Umweltbetriebsprüfungen mit ca. 1,8 Millionen Euro sowie 305 Umweltaudits mit ca. 3,4 Millionen Euro gefördert.

\section{Der Umweltpakt in Südtirol}

Inspiriert durch den Umweltpakt in Bayern hat die autonome italienische Region Südtirol im April 1998 den „Umweltpakt Südtirol“ abgeschlossen. Dieser Pakt ist eine Vereinbarung zwischen Politik, Verwaltung, Verbänden, Banken und Betrieben zur Umsetzung eines vorsorgenden betrieblichen Umweltschutzes. Es geht dabei nicht nur um die Vergabe von Fördergeldern seitens der öffentlichen Verwaltung, sondern auch um die Selbstverpflichtung zur aktiven und koordinierten Mitarbeit aller Institutionen.

Zur Sensibilisierung der Betriebe hat die federführende Landes-Umweltagentur unter anderem Pilotprojekte mit kleinen Betrieben oder über spezifische Themen durchgefiuhrt. Die Leistungen, die im Rahmen des Umweltpakts verwirklicht werden sollen, sind auf drei große Schwerpunkte verteilt (4):

\section{Tab. 1: Förderung von Umweltmanagemntsystemen in Sïdtirol}

\section{Verwendungszweck}

\section{Förderstufe 1:}

alle notwendigen Ausgaben für die Durchführung einer Umweltprüfung sowie die Erstellung einer Umweltpolitik und eines Umweltprogramms

\section{Förderstufe 2:}

alle notwendigen Ausgaben zur Einführung des Umweltmanagementsystems, zur Erstellung der Umwelterklärung und zur Begutachtung nach EG-Öko-Audit oder ISO 1400 I

\section{Förderhöhe}

$70-75 \%$ (KMU und Handwerksbetriebe)

$50 \%$ (große Industriebetriebe)

der förderfähigen Kosten

max. 10 Tage externe Beratung

$50 \%$ (KMU und Handwerksbetriebe)

$40 \%$ (große Industriebetriebe)

der förderfähigen Kosten

für ISO 14001 nur bei Abgabe eines Umweltberichtes
- Förderung von UMS als Herzstïck

- Förderung von Umweltinvestitionen,

- unterstïtzende Maßnahmen.

Die UMS-Förderung richtet sich besonders an KMU, da diese einerseits die Grundstruktur der Südtiroler - und allgemein der italienischen Wirtschaft darstellen, und anderseits im Vergleich $\mathrm{zu}$ größeren Betrieben einen höheren personellen und finanziellen Aufwand betreiben müssen, um ein UMS zu implementieren.

Wie die Tabelle zeigt, besteht das Förderprogramm aus zwei unabhängigen Förderstufen, die - ähnlich wie in Bayern - speziell auf KMU-Bedürfnisse ausgerichtet sind. Mit der ersten Förderstufe können sich diese Unternehmen an die mit EMAS verbundenen kulturellen und organisatorischen Veränderungen anpassen, ohne dass damit zusätzliche Kosten verbunden sind. Gerade die Veränderung der Unternehmenskultur in Richtung Eigenverantwortung und vorsorgender Einstellung zum Umweltschutz sind für einen Erfolg in KMU wichtig. An der zweiten Förderstufe interessant ist, dass Betriebe, die sich für eine Zertifizierung nach der internationalen Umweltmanagementnorm ISO 14001 entscheiden, nur dann den Förderbetrag erhalten, wenn sie auch einen Umweltbericht entsprechend der Vorgaben der Öko-Audit-Verordnung für die Umwelterklärung veröffentlichen. Außerdem wird die Verknüpfung mit anderen Managementsystemen im Sinne eines Total Quality Management propagiert.

Leider gibt es in Südtirol bisher noch sehr viel weniger Teilnehmer als in Bayern; insbesondere hat dieser neue Management-Ansatz im Tourismus mehr Erfolg als in anderen Bereichen.

\section{Anmerkungen}

(1) Vgl. dazu auch Petschow, U.: Kein Pakt für die Nachhaltigkeit. In: Ökologisches Wirtschaften, Nr. 1/1996, S. 4.

(2) Vgl. auch den Schwerpunkt „Integrierte Produktpolitik" der Ausgabe Nr. 6/2000 von Ökologisches Wirtschaften.

(3) Vgl. dazu näher www.umweltministerium.bayern.de (4) Vgl. z.B. Südtiroler Landesagentur für Umweltschutz und Arbeitssicherheit: Leitfaden „Einführung von Umweltmanagementsystemen für kleine Betriebe". Bozen 1998.

\section{Der Autor}

Giuseppe Santillo ist Politikwissenschaftler mit dem Schwerpunkt (Vergleich von) Umweltverwaltungen. Kontakt: Rheinsberger Str. 66, 10115 Berlin. Tel. 0163/5329099, E-Mail:

giuseppesantillo@hotmail.com 
(c) 20I0 Authors; licensee IÖW and oekom verlag. This is an article distributed under the terms of the Creative Commons Attribution Non-Commercial No Derivates License (http://creativecommons.org/licenses/by-nc-nd/3.o/), which permits unrestricted use, distribution, and reproduction in any medium, provided the original work is properly cited. 